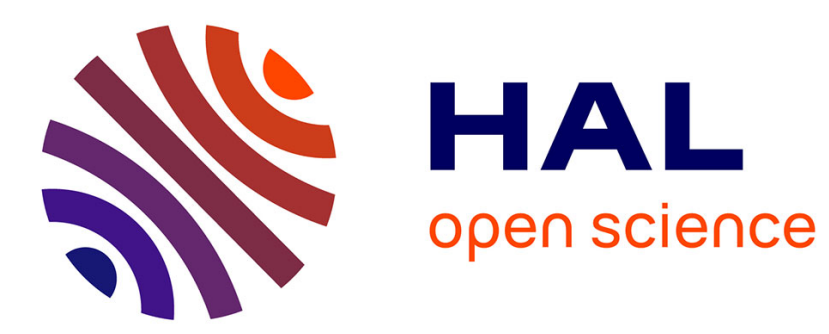

\title{
Sur les collapses de corps différentiels colorés en caractéristique nulle décrits par Poizat à l'aide des amalgames à la Hrushovski.
}

Thomas Blossier, Amador Martin-Pizarro

\section{To cite this version:}

Thomas Blossier, Amador Martin-Pizarro. Sur les collapses de corps différentiels colorés en caractéristique nulle décrits par Poizat à l'aide des amalgames à la Hrushovski.. 2008. hal-00261500

\author{
HAL Id: hal-00261500 \\ https://hal.science/hal-00261500
}

Preprint submitted on 7 Mar 2008

HAL is a multi-disciplinary open access archive for the deposit and dissemination of scientific research documents, whether they are published or not. The documents may come from teaching and research institutions in France or abroad, or from public or private research centers.
L'archive ouverte pluridisciplinaire HAL, est destinée au dépôt et à la diffusion de documents scientifiques de niveau recherche, publiés ou non, émanant des établissements d'enseignement et de recherche français ou étrangers, des laboratoires publics ou privés. 


\title{
SUR LES COLLAPSES DE CORPS DIFFÉRENTIELS COLORÉS EN CARACTÉRISTIQUE NULLE DÉCRITS PAR POIZAT À L'AIDE DES AMALGAMES À LA HRUSHOVSKI.
}

\author{
T. BLOSSIER ET A. MARTIN-PIZARRO*
}

\begin{abstract}
RÉsumÉ. Nous collapsons le corps différentiel rouge de Poizat en des corps différentiellement clos de rang de Morley $\omega \cdot 2$, chacun muni d'un sous-groupe additif définissable de rang $\omega$. En utilisant la dérivée logarithmique, on obtient un corps vert de rang $\omega \cdot 2$ avec un sous-groupe multiplicatif définissable divisible contenant le corps des constantes, qui reste définissable dans le réduit à la structure de corps vert.
\end{abstract}

Et ce qu'il advint.

\section{INTRODUCTION}

En 1991, Hrushovski [7, 5] donna une preuve de la conjecture de Mordell-Lang pour les corps de fonctions. Ce résultat était déjà connu en caractéristique nulle, mais l'originalité de cette nouvelle preuve réside dans son approche uniforme en toutes caractéristiques. Elle consiste à remplacer la structure du corps algébriquement clos de base, par une structure de corps dans laquelle l'énoncé de Mordell-Lang est équivalent à la notion modèle-théorique de monobasé pour un certain sous-groupe. En caractéristique nulle, il s'agit de montrer qu'un sous-groupe différentiel, le noyau de Manin, est monobasé. Il est alors utilisé un principe de dichotomie qui est satisfait par les géométries non triviales vivant dans un corps différentiel universel (appelé corps différentiellement clos) : ces géométries sont typiques de celle d'un corps algébriquement clos (dans ce cas, le corps des constantes) ou de celle d'un espace vectoriel. Au début des années 80, Zilber avait conjecturé ce principe de dichotomie pour toute géométrie associée à un ensemble fortement minimal. On rappelle qu'un ensemble fortement minimal est un ensemble irréductible de dimension $^{1} 1$.

Cette conjecture fut réfutée par Hrushovski [9] qui, par une méthode d'amalgamation utilisant des idées de Fraïssé , construisit des ensembles fortement minimaux avec des géométries plus exotiques. Cette méthode permit également à Poizat [14] de construire des corps $\omega$-stables en toutes caractéristiques munis d'un sous-groupe additif de rang commensurable (qu'il nomma corps rouges) ainsi qu'un corps en caractéristique nulle de rang $\omega \cdot 2$ muni d'un sous-groupe multiplicatif divisible sans

Date: 9 janvier 2008.

1991 Mathematics Subject Classification. Primary : 03C65; Secondary :03C50.

Key words and phrases. Model Theory, Differentially closed fields, Amalgamation method.

* Recherche effectuée avec le soutien du programme de recherche européen FP6-MOB-5-009541.

${ }^{1}$ Cette dimension est le rang de Morley. Une structure rangée par cette dimension est dite $\omega-$ stable. 
torsion définissable de rang $\omega$ (nommé vert). Associé audit procédé, le collapse consiste à effectuer l'amalgamation dans une classe restreinte afin de forcer certains types à devenir algébriques. En conséquence, Poizat conjectura l'existence de collapses de rang fini autant pour les corps rouges en caractéristique positive que pour le corps vert. Les premiers furent collapsés dans [3], ce qui montra l'existence de corps de rang fini en caractéristique positive qui ne sont pas additivement minimaux. Dans [1], les corps verts furent également collapsés, ce qui montra l'existence de mauvais corps en caractéristique nulle. Rappelons au lecteur qu'un corps de rang fini est mauvais s'il admet un sous-groupe propre multiplicatif définissable divisible et que la non-existence de tels corps avait été conjecturée originellement dans le programme de classification de groupes de rang de Morley fini. En caractéristique positive, l'existence d'un mauvais corps forcerait à avoir une finitude de nombres premiers de Mersenne en $p$, c'est à dire de la forme $\frac{p^{n}-1}{p-1}$ [16].

Notons qu'un corps de rang de Morley fini en caractéristique nulle est additivement minimal. Néanmoins dans [14], Poizat conjectura qu'il devait être également possible d'obtenir des collapses rouges de rang minimal, c'est à dire de rang $\omega \cdot 2$. Rappelons que sa construction d'un corps rouge de caractéristique nulle, qui est de rang $\omega^{2} \cdot 2$, est obtenue par amalgamation de corps différentiels. Le but de cet article est de collapser ce corps. Pour cela, nous suivrons la méthode des collapses de corps rouges de caractéristique positive [3]. Par contre, la théorie des corps différentiellement clos, même si elle est $\omega$-stable, présente des particularités qui nous ont obligés à être minutieux dans la susdite adaptation. Les deux points délicats résident d'une part dans le fait que le sous-groupe additif rouge est ici un espace vectoriel sur un corps flottant, le corps des constantes, et d'autre part dans le fait que les rangs dans la théorie des corps différentiellement clos ne coïncident pas. Nous devons alors estimer le rang de Morley en utilisant l'encadrement donné par le degré différentiel pour les types à collapser. Ces types correspondent aux types génériques des sous-groupes additifs différentiels pour lesquels nous pouvons décrire uniformément leurs rangs, ceci à l'aide d'un résultat de Pillay et Pong [12].

La structure de cet article est la suivante : nous commençons par rappeler la construction par amalgamation de Poizat. Nous explicitons ensuite le calcul des rangs dans cette théorie. Cette description technique est nécessaire pour coder les types à collapser dans la partie qui suit. Dans ces deux parties, nous rappelons également les résultats de la théorie des corps différentiellement clos qui sont utilisés. La partie suivante décrit les collapses et leurs axiomatisations. Finalement, nous regardons le corps vert obtenu par dérivée logarithmique.

Tout au long de cet article, une certaine familiarité avec le procédé d'amalgamation et en particulier avec les constructions de corps colorés sera requise (voir par exemple $[14,2,3])$.

Les auteurs aimeraient remercier B. Poizat qui a inspiré ce travail ainsi que A. Pillay, F.O. Wagner et M. Ziegler pour leurs commentaires avisés.

\section{Poizht et les rouges}

Dans cette partie, nous rappelons au lecteur certains des résultats de [14] que nous utiliserons pour le collapse. Commençons par fixer les notations : nous travaillerons dans un corps différentiellement clos universel $\mathbb{K}$ de caractéristique nulle et nous noterons $C$ le corps des constantes (c.à.d. les éléments de dérivée nulle). 
Soit $L:=\{0,+,-, \cdot, d\}$ le langage des anneaux différentiels. Nous formons le langage $L^{*}$ en ajoutant à $L$ un prédicat unaire $\mathrm{R}$ et pour toute $L^{*}$-structure, les points dans $\mathrm{R}$ seront nommés rouges, les autres blancs. Dans la suite de ce papier, nous considérons uniquement des $L^{*}$-structures $k$ telles que la restriction de $k$ au langage $L$ soit un sous-corps différentiel de $\mathbb{K}$ et telles que $\mathrm{R}(k)$ soit un sous-espace vectoriel de $(k,+) \operatorname{sur} C_{k}(:=C \cap k)$. On appellera ces structures corps différentiels rouges. Nous appellerons degré différentiel d'un corps différentiel la taille d'une base de transcendance différentielle de ce corps. Nous définissons alors sur les corps différentiels rouges $k$ de degré différentiel fini la prédimension suivante :

$$
\delta(k)=2 \text { deg.dif } k-\operatorname{dim} \cdot \operatorname{lin}_{\mathrm{C}} \mathrm{R}(k)
$$

où deg.dif $k$ est le degré différentiel de $k$ et $\operatorname{dim} \cdot \operatorname{lin}_{\mathrm{C}} \mathrm{R}(k)$ la dimension linéaire des points rouges sur $C$.

Notons que $C$ dépend du sous-corps différentiel considéré, mais du fait que l'indépendance linéaire sur les constantes s'exprime à l'aide du Wronskien, la dimension linéaire des points rouges reste inchangée qu'on la considère au-dessus de $C_{k}$ ou au-dessus de l'ensemble des constantes $C$. Nous utiliserons donc cette notation ambiguë pour $\delta$.

Notons $\mathcal{K}_{0}$ la classe élémentaire des corps $k$ différentiels algébriquement clos rouges, dont tout sous-corps différentiel de degré différentiel fini a une prédimension positive ou nulle. (Cette classe est $\forall \exists$-axiomatisable.)

Pour tout corps $k \subset \mathbb{K}$ et toute partie $A \subset \mathbb{K}$, on notera $k\langle A\rangle$ la clôture algébrique du corps différentiel engendré par $A$ au-dessus de $k$. On dira que $A$ engendre $k^{\prime}$ audessus de $k$ si $k^{\prime}=k\langle A\rangle$.

Pour tout uple fini $\bar{a}$ et tout partie $B$ dans un corps $k$ de $\mathcal{K}_{0}$, on utilisera la notation :

$$
\delta(\bar{a} / B):=2 \operatorname{deg} \cdot \operatorname{dif}(\bar{a} / B)-\operatorname{dim} \cdot \operatorname{lin}_{\mathrm{C}}(\mathrm{R}(\mathbb{Q}\langle\bar{a} B\rangle) / \mathrm{R}(\mathbb{Q}\langle B\rangle)) .
$$

Cette prédimension n'est pas sous-modulaire : l'inégalité $\delta(\bar{a} / B) \leq \delta(\bar{a} / \mathbb{Q}\langle\bar{a}\rangle \cap$ $\mathbb{Q}\langle B\rangle)$ n'est pas toujours vérifiée du fait que le corps des constantes n'est pas fixe. Par contre, dans le cas où $\mathbb{Q}\langle\bar{a}\rangle, \mathbb{Q}\langle B\rangle$ et $\mathbb{Q}\langle\bar{a} B\rangle$ ont même corps de constantes, cette inégalité est bien vérifiée.

On définit de la manière habituelle la notion d'autosuffisance : soient $k \subset k^{\prime}$ deux corps dans $\mathcal{K}_{0}$; on dit que $k$ est autosuffisant dans $k^{\prime}$ ou que $k^{\prime}$ est une extension autosuffisante de $k$ (noté $\left.k \leq k^{\prime}\right)$ si $\delta(\bar{a} / k) \geq 0$ pour tout uple fini $\bar{a}$ de $k^{\prime}$.

Notons que $k$ est autosuffisant dans $k^{\prime}$ si et seulement si les points rouges de $k\left\langle C_{k^{\prime}}\right\rangle$ sont engendrés par les points rouges de $k$ et $k\left\langle C_{k^{\prime}}\right\rangle$ est autosuffisant dans $k^{\prime}$ [14, Lemme 2.3.1].

On dira par la suite qu'un ensemble $A$ d'un corps $k^{\prime}$ de $\mathcal{K}_{0}$ est autosuffisant dans $k^{\prime}$ si $\mathbb{Q}\langle A\rangle$ est autosuffisant dans $k^{\prime}$ et $A$ engendre les points rouges de $\mathbb{Q}\langle A\rangle$ comme espace vectoriel sur $C_{\mathbb{Q}\langle A\rangle}$.

La relation d'autosuffisance est transitive pour la classe $\mathcal{K}_{0}$ mais il n'existe pas de clôture autosuffisante uniquement déterminée, elle flotte avec les constantes: soit $K$ un corps de $\mathcal{K}_{0}$, alors pour tout sous-corps $k$ de $K$ il existe un plus petit sous-corps autosuffisant de $K$ contenant $C_{K} \cup k$. Si $k_{1}$ et $k_{2}$ sont deux corps de $\mathcal{K}_{0}$ contenant $k$, autosuffisants dans $K$ et de degré différentiel minimal au-dessus de $k$ alors ils sont engendrés par des bases linéaires rouges qui se correspondent par un isomorphisme linéaire au-dessus des constantes globales. 
Dans [14], Poizat amalgame la classe $\mathcal{K}_{0}$ par la méthode de Hrushovski-Fraïssé : Soient $k_{1}$ et $k_{2}$ deux corps de $\mathcal{K}_{0}$ d'intersection $k$. On place $k_{1}$ et $k_{2}$ de façon linéairement disjointe au-dessus de $k$ et on considère la clôture algébrique $k^{\prime}=$ $k\left\langle k_{1} \cup k_{2}\right\rangle$ du corps différentiel engendré par $k_{1}$ et $k_{2}$. (Notons que le corps des constantes de $k^{\prime}$ peut être plus gros que la clôture algébrique des corps de constantes de $k_{1}$ et $k_{2}$.) On colorie en rouge les points de $k^{\prime}$ engendrés par les points rouges de $k_{1}$ et de $k_{2}$. La structure ainsi obtenue s'appelle l'amalgame libre de $k_{1}$ et $k_{2}$ au-dessus de $k$. Elle appartient à $\mathcal{K}_{0}$ (voir [14, Lemme 2.3.3]).

Théorème 2.1. [14] La sous-classe de $\mathcal{K}_{0}$ consistant en les corps différentiels de degré différentiel fini a la propriété d'amalgamation respectant les plongements autosuffisants (donnée par l'amalgame libre). Soit $\mathbb{K}_{\omega}$ sa limite de Frä̈ssé. Alors $\mathbb{K}_{\omega}$ est à isomorphisme près l'unique modèle dénombrable $\omega$-saturé de sa théorie, cette théorie est $\omega$-stable de rang $\omega^{2} \cdot 2$, et les rouges forment un sous-groupe additif de $\operatorname{rang} \omega^{2}$.

Pour la suite, on appelle théorie $\mathrm{DCF}_{0}$ colorée la théorie de $\mathbb{K}_{\omega}$. (La notation $\mathrm{DCF}_{0}$ seule désignera la théorie des corps différentiellement clos de caractéristiques nulles dans le langage $L$.) Le calcul des rangs (de Lascar et de Morley) dans $\mathrm{DCF}_{0}$ colorée se fait, comme habituellement, en décomposant les extensions autosuffisantes en tours d'extensions minimales (extensions autosuffisantes ne contenant pas de sous-extensions autosuffisantes propres intermédiaires).

Il est facile de vérifier qu'une extension minimale correspond à l'une des quatre possibilités suivantes. Par contre, le calcul des rangs des extensions minimales de second type est assez technique (voir la section suivante) :

Proposition 2.2. Une extension minimale $k^{\prime}$ dans $\mathcal{K}_{0}$ d'un corps $k$ dans $\mathcal{K}_{0}$ correspond à l'un des cas suivants et on obtient les rangs suivants si on suppose de plus $k^{\prime}$ plongé de manière autosuffisante dans $\mathbb{K}_{\omega}$ :

(1) $k^{\prime}$ est engendré par un point blanc différentiellement algébrique sur $k$. Dans ce cas $\delta\left(k^{\prime} / k\right)=0$ et les rangs (de Lascar et de Morley dans $\mathrm{DCF}_{0}$ colorée) du point blanc sur $k$ sont finis.

Sinon on a $C_{k^{\prime}}=C_{k}$ et ou bien :

(2) $k^{\prime}$ est engendré par une base $\bar{a}$ de $2 n$ points rouges linéairement indépendants et $k^{\prime}$ est de degré différentiel $n$ sur $k$. Dans ce cas $\delta\left(k^{\prime} / k\right)=0$ et les rangs de $\bar{a}$ sur $k$ sont inférieurs à $\omega \cdot 2$;

(3) $k^{\prime}$ est engendré par un point rouge différentiellement transcendant sur $k$. Dans ce cas $\delta\left(k^{\prime} / k\right)=1$ et les rangs de ce point rouge générique sont égaux $\omega^{2}$.

(4) $k^{\prime}$ est engendré par un point blanc différentiellement transcendant sur $k$. Dans ce cas $\delta\left(k^{\prime} / k\right)=2$ et les rangs de ce point blanc générique sur $k$ sont égaux $\omega^{2} \cdot 2$.

\section{RANGS COLORÉS}

Pour collapser les corps différentiels rouges, il est nécessaire d'analyser précisément le rang des extensions minimales de second type dans la proposition 2.2. Pour cela, commençons par rappeler des résultats sur la théorie $\mathrm{DCF}_{0}$ et plus généralement de stabilité qui seront utiles.

Pour la suite, nous noterons RU (resp. RU*) le rang de Lascar, RM et dM (resp. $\mathrm{RM}^{*}$ et $\mathrm{dM}^{*}$ ) le rang et degré de Morley, dans la théorie $\mathrm{DCF}_{0}$ (resp. dans 
la théorie $\mathrm{DCF}_{0}$ colorée). Nous noterons dcl, acl et $\downarrow$ la clôture définissable, la clôture algébrique, et l'indépendance dans la théorie $\mathrm{DCF}_{0}$.

Fait 3.1. Dans $\mathrm{DCF}_{0}$ :

(1) Soit $\bar{a}$ un uple d'éléments différentiellement algébriques sur $B$. Alors $\operatorname{RM}(\bar{a} / B) \leq$ $\operatorname{RD}(\bar{a} / B)$ où $\operatorname{RD}(\bar{a} / B)$ (le rang différentiel de $\bar{a}$ sur $B$ ) correspond au degré de transcendance du corps différentiel engendré par $\bar{a}$ au dessus de celui engendré par $B$.

(2) Tout type $p$ vérifie,

$$
\omega \cdot \operatorname{deg} \cdot \operatorname{dif}(p) \leq \mathrm{RU}(p) \leq \mathrm{RM}(p)<\omega \cdot(\operatorname{deg} \cdot \operatorname{dif}(p)+1)(\operatorname{voir}[4]) .
$$

(3) Tout sous-groupe additif définissable de $\mathbb{K}^{n}$ est connexe (car $\mathbb{K}$ est de caractéristique nulle) et est un sous-espace vectoriel sur les constantes (voir par exemple [17, Corollary 1.12]).

(4) Les rangs de Lascar et de Morley d'un groupe définissable sont égaux [12].

(5) Pour tout uple $\bar{a}$ de taille $n$, tout uple $\bar{b}$ et tout ensemble de paramètres $B$ tels que deg.dif $(\bar{a} / B)=n$ (c.à.d $\bar{a}$ générique sur $B$ ) et $\bar{b}$ différentiellement algébrique sur $\bar{a} B$, alors il existe $\bar{c}$ différentiellement algébrique sur $B$ telle que $\operatorname{RU}(\bar{a} \bar{b} / B \bar{c})=\omega \cdot n$ et $\operatorname{RM}(\bar{c} / B) \leq \operatorname{RM}(\bar{b} / \bar{a} B)$.

Notons alors que dans $\mathrm{DCF}_{0}$ :

- Si $p$ est un type sur $B$ tel que $\mathrm{RU}(p)=\omega \cdot n$ alors une réalisation $\bar{a}$ de $p$ est générique sur $A \supset B$ si et seulement si $\operatorname{deg} \cdot \operatorname{dif}(\bar{a} / A)=n$.

- Si $\varphi(\bar{x}, \bar{b})$ est une formule telle que $\operatorname{RM}(\varphi)=\omega \cdot n$ alors une réalisation $\bar{a}$ de $\varphi$ est générique sur $B \supset \bar{b}$ si et seulement si $\operatorname{deg} \cdot \operatorname{dif}(\bar{a} / B)=n$.

- Soit $p$ un type sur $B$ de rang de Lascar $\omega \cdot n$. Par les inégalités de Lascar, pour toute réalisation $\bar{a}$ de $p$ et tout ensemble $A$ d'éléments différentiellement algébriques sur $B$, on a $\bar{a} \underset{B}{\downarrow} A$. De plus les corps $\mathbb{Q}\langle\bar{a} B\rangle$ et $\mathbb{Q}\langle B\rangle$ ont même corps de constantes.

Quelques mots sur (5) (nous remercions A. Pillay pour nous avoir communiqué l'argument) : soit $p$ le type générique sur $B$ d'un élément différentiellement transcendant dans $\mathrm{DCF}_{0}$. Le $p$-poids de $\bar{a} \bar{b}$ sur $B$ est $n$. Soit $Y$ l'ensemble des uples $\bar{c}$ définissables sur $\bar{a} \bar{b} B$ de $p$-poids nul sur $B$. Par [11, Corollary 7.1.20]), le type fort de $\bar{a} \bar{b}$ sur $B Y$ est équivalent à $p^{(n)}$ et donc de $p$-poids $n$. Donc il existe $\bar{c}$ dans $Y$ différentiellement algébrique sur $B$ tel que $\operatorname{RU}(\bar{a} \bar{b} / B \bar{c})=\omega \cdot n$. De plus, $\operatorname{RM}(\bar{c} / B)$ est borné par $\operatorname{RM}(\bar{b} / \bar{a} B)$ (car $\bar{c}$, ayant un rang fini, il ne peut devier avec $\bar{a}$ sur $B$.)

Nous aurons également besoin du lemme suivant [18] que nous appliquerons aux groupes additifs $\mathbb{G}_{a}^{n}\left(\right.$ où $\left.\mathbb{G}_{a}:=(\mathbb{K},+)\right)$ :

Lemme 3.2. Soient $G$ un groupe $\omega$-stable abélien, et $a, b$ et $c$ des éléments de $G$ deux à deux indépendants sur $B$ tels que $a=b+c$. Alors :

(1) Leurs types forts sur $B$ ont même stabilisateur $U$ qui est connexe et définissable sur $\operatorname{acl}^{\text {eq }}(B)$.

(2) $a, b$ et $c$ sont des éléments génériques de cossettes de $U$ définissables sur $\operatorname{acl}^{e q}(B)$. 
Revenons maintenant au calcul des rangs des extensions minimales.

Un point blanc différentiellement algébrique a un type de nature purement corpique. Ses rangs dans $\mathrm{DCF}_{0}$ coloré correspondent donc aux rangs dans $\mathrm{DCF}_{0}$ sans couleur; ses rangs sont donc finis.

Le second cas est beaucoup plus délicat : notons $\bar{a}$ une base linéaire de $\mathrm{R}\left(k^{\prime}\right)$ au dessus de $k$. Pour commencer supposons que $\operatorname{RU}(\bar{a} / k)=\omega \cdot n$ où $n=\operatorname{deg} \cdot \operatorname{dif}(\bar{a} / K)$. (En utilisant le fait 3.1 (5), on pourra ensuite se ramener à cette situation en ajoutant un uple fini d'éléments différentiellement algébriques, qui sont de rangs finis). Avec cette hypothèse, une réalisation $\bar{a}^{\prime}$ de $p$ est indépendante de $B$ si et seulement si deg.dif $\left(\bar{a}^{\prime} / B k\right)=n$. Par minimalité, $\delta(\bar{a} / k \cup M \cdot \bar{a})<0$ pour toute matrice $M$ de $\mathrm{M}_{2 n}\left(C_{k}\right)$ de rang $1 \leq d<2 n$, c'est-à-dire $2 \operatorname{deg}$.dif $(\bar{a} / k \cup M \cdot \bar{a})<$ $2 n-d$. Du fait que la clôture différentielle de $k$ n'a pas plus de constantes que celles de $k$ et que dans $\mathrm{DCF}_{0}, \bar{a}$ est indépendant des constantes au-dessus de $k$, on en déduit que 2 deg. $\operatorname{dif}(\bar{a} / k \cup M \cdot \bar{a})<2 n-d$ pour toute matrice $M$ de $\mathrm{M}_{2 n}(C)$ de rang $d$ parce que $\bar{a}$ et $C$ sont en position d'héritier-cohéritier au-dessus de la clôture différentielle de $k$.

Considérons alors une formule $\varphi$ (à paramètres $\bar{b} \in k$ ) de $\mathrm{DCF}_{0}$ qui isole $p$ des types de rangs de Morley supérieurs ou égaux. De plus, on peut supposer que $\varphi$ exprime d'une part l'indépendance linéaire de $\bar{a}$ sur les constantes et d'autre part la minimalité, c.à.d. que pour toute uple $\bar{a}^{\prime}$ dans $\varphi$, pour tout $0<d<2 n$, pour toute matrice $M$ de $\mathrm{M}_{2 n}(C)$ de rang $d$, le degré différentiel de $\bar{a}^{\prime}$ sur $\bar{b} \cup M \cdot \bar{a}^{\prime}$ est strictement inférieur à $n-d / 2$. (Ce deuxième point s'obtient par compacité.)

Lemme 3.3. Soit $\varphi(\bar{x}, \bar{b})$ une formule comme ci-dessus, c'est-à-dire telle que $|x|=$ $2 n$, toute réalisation $\bar{a}^{\prime}$ de $\varphi(\bar{x}, \bar{b})$ est linéairement indépendante sur les constantes, deg.dif $(\varphi(\bar{x}, \bar{b}))=n$ et $\operatorname{deg} \cdot \operatorname{dif}\left(\bar{a}^{\prime} / \bar{b} \cup M \cdot \bar{a}^{\prime}\right)<n-d / 2$ pour toute matrice $M \in$ $\mathrm{M}_{2 n}(C)$ de rang $d$ où $0<d<2 n$. Soit $K \in \mathcal{K}_{0}$ tel que $\bar{b} \in K$. Si $\bar{a}^{\prime}$ est une réalisation rouge de $\varphi$ alors $\delta\left(\bar{a}^{\prime} / K\right) \leq 0$. De plus $\delta\left(\bar{a}^{\prime} / K\right)=0$ si et seulement si $\bar{a}^{\prime}$ est dans le $C$-espace vectoriel engendré par $\mathrm{R}(K)$ ou $\bar{a}^{\prime}$ est $C$-linéairement indépendant au dessus de $\mathrm{R}(K)$ et de degré différentiel $n$ sur $K$.

Démonstration. Soit $d=2 n-\operatorname{dim} \cdot \operatorname{lin}_{\mathrm{C}}\left(\bar{a}^{\prime} / R(K)\right)$. Soit $M$ de rang $d$ telle que $M \cdot \bar{a}^{\prime} \in \mathrm{R}(K)$. Si $0<d<2 n$, alors 2 deg.dif $\left(\bar{a}^{\prime} / K\right) \leq 2 \operatorname{deg} \cdot \operatorname{dif}\left(\bar{a}^{\prime} / \bar{b} \cup M \cdot \bar{a}^{\prime}\right)<$ $2 n-d=\operatorname{dim}^{-} \operatorname{lin}_{\mathrm{C}}\left(\bar{a}^{\prime} / R(K)\right)$ et donc $\delta\left(\bar{a}^{\prime} / K\right)<0$. Si $d=0$, alors $\delta\left(\bar{a}^{\prime} / K\right) \leq 0$ car deg.dif $(\varphi(\bar{x}, \bar{b}))=n$; et $\delta\left(\bar{a}^{\prime} / K\right)=0$ si et seulement si $\operatorname{deg} \cdot \operatorname{dif}\left(\bar{a}^{\prime} / K\right)=n$.

Comme $k$ est autosuffisant, le type $p^{*}$ de $\bar{a}$ sur $k$ dans $\mathrm{DCF}_{0}$ colorée est déterminé par $p$ et la formule " $\bar{a}$ est rouge". On peut supposer de plus l'ensemble des paramètres $\bar{b}$ autosuffisant et considérer pour la suite que $p$ et $p^{*}$ sont les types de $\bar{a}$ sur $\bar{b}$ respectivement dans $\mathrm{DCF}_{0}$ et $\mathrm{DCF}_{0}$ colorée.

Lemme 3.4. Le type $p^{*}$ est régulier et a une géométrie localement modulaire.

(1) Si $p^{*}$ est non trivial alors $p$ est le type générique d'un sous-C-espace affine de $\mathbb{K}^{2 n}$ et $\operatorname{RU}^{*}(\bar{a} / k)=\operatorname{RM}^{*}(\bar{a} / k)=\omega$.

(2) Si $p^{*}$ est trivial alors $\operatorname{RU}^{*}(\bar{a} / k) \leq \operatorname{RM}^{*}(\bar{a} / k)<\omega$.

Démonstration. Du fait que $\mathrm{RU}(p)=\omega \cdot n$ et donc que l'on réalise une extension non déviante de $p$ si et seulement si on reste de degré différentiel $n$, en appliquant le lemme 3.3 , on en déduit que si $\bar{a}, \bar{a}_{1} \ldots \bar{a}_{m}$ sont des réalisations de $p^{*}$, alors $\bar{a}$ dévie sur $\bar{a}_{1} \ldots \bar{a}_{m}$ au-dessus de $k$ si et seulement si $\bar{a}$ est dans l'espace vectoriel engendré par $\bar{a}_{1} \ldots \bar{a}_{m}$. Donc $p^{*}$ est régulier et sa géométrie est localement modulaire. 
(1) Supposons $p^{*}$ non trivial. Alors il est facile de vérifier qu'il existe une réalisation $\bar{a}_{1}$ de $p$ et deux uples $\bar{a}_{2}$ et $\bar{a}_{3}$ de $\mathbb{K}^{2 n}$ deux à deux indépendants sur $\bar{b}$ dans $\mathrm{DCF}_{0}$ tels que $\bar{a}_{1}=\bar{a}_{2}+\bar{a}_{3}$. Par le lemme $3.2, \bar{a}_{1}$ est générique d'une cossette d'un sous-groupe additif de $\mathbb{K}^{2 n}$ définissable dans $\mathrm{DCF}_{0}$. Par le fait 3.1 (3) et (4) ce sousgroupe est un sous-espace vectoriel sur les constantes et $\operatorname{RM}(p)=\mathrm{RU}(p)=\omega \cdot n$. Alors $\varphi$ est de rang $\omega \cdot n$ et de degré 1 . Soit $\bar{a}^{\prime}$ rouge satisfaisant $\varphi$ et $B$ un ensemble de paramètres autosuffisant contenant $\bar{b}$. Comme $B$ est autosuffisant, $\delta\left(\bar{a}^{\prime} / B\right)=0$. Donc par le lemme 3.3, ou bien $\bar{a}^{\prime}$ tombe dans le $C$-espace vectoriel engendré par $B$ et est donc de rang fini sur $B$ ou bien $\bar{a}^{\prime}$ réalise l'unique extension non déviante de $p^{*}$ à $B$. D'où $\mathrm{RM}^{*}\left(p^{*}\right) \leq \omega$ et comme $p^{*}$ a une géométrie d'espace affine sur $C$, les rangs sont exactement $\omega$.

(2) Supposons $p^{*}$ trivial. Soit $\bar{a}$ une réalisation d'une extension déviante $q^{*}$ de $p^{*}$ sur un ensemble $B$. Par stabilité de la théorie colorée, la base canonique de $q^{*}$ sur $B$ est définissable sur une suite de Morley de ce type, suite qu'on peut supposer indépendante de $\bar{a}$ au-dessus de $B$. Par trivialité de $p^{*}$, il existe un élement $\bar{a}^{\prime}$ de cette suite, tel que $\bar{a}$ dévie sur $\bar{a}^{\prime}$ au-dessus de $\bar{b}$ et donc $\bar{a}$ est dans l'espace vectoriel engendré par $\bar{a}^{\prime} \bar{b}$ par le lemme 3.3. D'où $\operatorname{RU}^{*}\left(q^{*}\right) \leq \operatorname{RU}^{*}\left(\bar{a} / \bar{a}^{\prime} \bar{b}\right) \leq 2 n(2 n+|\bar{b}|)$. Donc $p^{*}$ est de rang de Lascar fini. En revanche, on ne peut pas conclure par le même argument pour le rang de Morley car le rang de $\varphi$ dans $\mathrm{DCF}_{0}$ n'est pas nécessairement monomial. Remarquons que $\bar{a}$ peut se décomposer en deux uples $\bar{a}_{0}$ et $\bar{a}_{1}$ de taille $n$, avec $\bar{a}_{1}$ différentiellement algébrique sur $\bar{a}_{0} \bar{b}$. On peut alors également supposer que $\operatorname{RD}\left(\bar{a}_{1}^{\prime} / \bar{a}_{0}^{\prime} \bar{b}\right) \leq \mathrm{RD}\left(\bar{a}_{1} / \bar{a}_{0} \bar{b}\right)$ pour toute réalisation $\bar{a}^{\prime}=\bar{a}_{0}^{\prime} \bar{a}_{1}^{\prime}$ de $\varphi$.

Par ailleurs $p^{*}$ trivial signifie que pour toutes réalisations rouges $\bar{a}, \bar{a}^{\prime}$ et $\bar{a}^{\prime \prime}$ de $p$, si deg.dif $\left(\bar{a} \bar{a}^{\prime} \bar{a}^{\prime \prime} / \bar{b}\right)=3 n$ alors $\bar{a}^{\prime}+\bar{a}^{\prime \prime}-\bar{a}$ n'est pas une réalisation de $p$. Par compacité, il existe une formule $\psi$ dans $p$ tel que si $\bar{a}, \bar{a}^{\prime}$ et $\bar{a}^{\prime \prime}$ rouges satisfont $\psi$ et deg. $\operatorname{dif}\left(\bar{a} \bar{a}^{\prime} \bar{a}^{\prime \prime} / \bar{b}\right)=3 n$, alors $\bar{a}^{\prime}+\bar{a}^{\prime \prime}-\bar{a}$ ne satisfait pas $\psi$. On peut supposer que $\varphi \rightarrow \psi$. Alors pour tout type $q$ dans $\varphi$ de $\operatorname{RU}(q)=\omega \cdot n$, le type $q^{*}$ (c.à.d. $q$ et rouge) a une géométrie triviale. Notons que cette propriété peut être imposée uniformément sur les paramètres. On appelle alors une telle formule $\varphi$ une formule à géométries triviales.

On va maintenant montrer par induction que toute réalisation rouge d'une telle formule a un rang de Morley fini dans $\mathrm{DCF}_{0}$ colorée :

Sous-lemme. Soit $\varphi(\bar{x}, \bar{b})$ vérifiant les propriétés ci-dessus (avec $|x|=2 n$ et $\bar{b}$ autosuffisant). Pour tout $\bar{a}$ rouge satisfaisant $\varphi(\bar{x}, \bar{b})$, le rang $\operatorname{RM}^{*}(\bar{a} / \bar{b})$ est fini et borné en fonction de $n,|\bar{b}|, \operatorname{RD}\left(\bar{a}_{1} / \bar{a}_{0} \bar{b}\right)$ et $l$ tel que $\operatorname{RM}(\bar{a} / \bar{b}) \leq \omega \cdot n+l$.

Démonstration du sous-lemme. Soit $\bar{a}$ rouge satisfaisant $\varphi(\bar{x}, \bar{b})$. On considère une formule $\psi(\bar{x}, \bar{b})$ impliquant $\varphi$ et isolant le type $q$ de $\bar{a}$ sur $\bar{b}$ des types de rangs de Morley supérieurs ou égaux. On fait alors la preuve par induction sur $\operatorname{RD}\left(\bar{a}_{1} / \bar{a}_{0} \bar{b}\right)$ et $\operatorname{RM}(\psi(\bar{x}, \bar{b}))$.

Tout d'abord, si $\operatorname{RD}\left(\bar{a}_{1} / \bar{a}_{0} \bar{b}\right)=0$, alors $\bar{a}_{1}$ est algébrique sur $\bar{a}_{0} \bar{b}$ et donc

- ou bien $\operatorname{RM}(\bar{a} / \bar{b})<\omega \cdot n$ (c.à.d. $\operatorname{deg} . \operatorname{dif}(\bar{a} / \bar{b})<n)$ et alors par le lemme $3.3, \bar{a}$ est dans l'espace vectoriel engendré par $\bar{b}$. On conclut que $\operatorname{RM}^{*}(\bar{a} / \bar{b}) \leq 2 n|\bar{b}|$.

- ou bien $\operatorname{RM}(\bar{a} / \bar{b})=\omega \cdot n$ et $\operatorname{RU}(\bar{a} / \bar{b})=\omega \cdot n$. Alors pour tout $\bar{a}^{\prime}$ rouge satisfaisant $\psi$ et tout ensemble $B \supset \bar{b}$ autosuffisant, ou bien $\bar{a}^{\prime}$ réalise une extension non déviante à $B$ de $q^{*}$ ou bien $\bar{a}^{\prime}$ n'est pas indépendant de $B$ au-dessus de $\bar{b}$. Dans ce deuxième cas, par trivialité de $q^{*}$, il existe alors une réalisation $\bar{a}^{\prime \prime}$ de $q^{*}$ 
déviante avec $\bar{a}^{\prime}$ au-dessus de $\bar{b}$ telle que $\operatorname{RM}^{*}\left(\bar{a}^{\prime} / B\right) \leq \operatorname{RM}^{*}\left(\bar{a}^{\prime} / \bar{b} \bar{a}^{\prime \prime}\right)$. Alors $\bar{a}^{\prime}$ tombe dans l'espace vectoriel engendré par $\bar{a}^{\prime \prime} \bar{b}$ et donc $\operatorname{RM}^{*}\left(\bar{a}^{\prime} / \bar{a}^{\prime \prime} \bar{b}\right) \leq$ $2 n(2 n+|\bar{b}|)$. D'où on déduit que $\mathrm{RM}^{*}(\bar{a} / \bar{b}) \leq 2 n(2 n+|\bar{b}|)+1$.

Si $\operatorname{RD}\left(\bar{a}_{1} / \bar{a}_{0} \bar{b}\right)=i+1$. Le cas $\operatorname{RM}(\psi(\bar{x}, \bar{b})) \leq \omega \cdot n$ se traite comme décrit ci-dessus. Sinon, $\omega \cdot n<\operatorname{RM}(\psi(\bar{x}, \bar{b})) \leq \omega \cdot n+k$ et on distingue alors deux cas :

- Ou bien $\operatorname{RU}(\bar{a} / \bar{b})=\omega \cdot n$. D'une part, par induction sur le rang de Morley, l'ensemble des $\operatorname{RM}^{*}\left(\bar{a}^{\prime} / \bar{b}\right)$ pour les réalisations rouges $\bar{a}^{\prime}$ de $\psi$ telles que $\operatorname{RM}\left(\bar{a}^{\prime} / \bar{b}\right)<\operatorname{RM}(\psi(\bar{x}, \bar{b}))$ est uniformément borné. D'autre part si $\bar{a}^{\prime}$ est une réalisation rouge générique de $\psi$ qui dévie sur un ensemble autosuffisant $B$ contenant $\bar{b}$, alors comme précédemment, $\operatorname{RM}^{*}\left(\bar{a}^{\prime} / B\right) \leq 2 n(2 n+|\bar{b}|)$. On en déduit une borne pour $\operatorname{RM}^{*}(\bar{a} / \bar{b})$.

- Ou bien $\operatorname{RU}(\bar{a} / \bar{b})>\omega \cdot n$. Alors, par le Fait 3.1 (5) il existe un élément blanc $c$ différentiellement algébrique sur $\bar{b}$, tel que $\operatorname{RM}(\bar{a} / \bar{b} c)<\operatorname{RM}(\bar{a} / \bar{b})$ et $\operatorname{RM}(c / \bar{b}) \leq \operatorname{RM}\left(\bar{a}_{1} / \bar{a}_{0} \bar{b}\right)$. Alors $\operatorname{RD}\left(\bar{a}_{1} / \bar{a}_{0} \bar{b} c\right)<\operatorname{RD}\left(\bar{a}_{1} / \bar{a}_{0} \bar{b}\right)$ et donc par hypothèse d'induction $\operatorname{RM}^{*}(\bar{a} / \bar{b} c)$ est fini et borné en fonction de $|\bar{b}|+1$, $\operatorname{RD}\left(\bar{a}_{1} / \bar{a}_{0} \bar{b} c\right) \leq i$ et $\operatorname{RM}(\bar{a} / \bar{b} c) \leq \operatorname{RM}(\bar{a} / \bar{b})$. On considère alors $\theta(\bar{x}, z, \bar{b})$ contenant $\psi$, satisfaite par $\bar{a} c$ et telle que $\operatorname{RD}\left(\bar{x}_{1} / \bar{x}_{0} \bar{b} z\right) \leq i$. En particulier il existe une borne uniforme finie de $\operatorname{RM}^{*}\left(\bar{a}^{\prime} / \bar{b} c^{\prime}\right)$, pour toute réalisation $\bar{a}^{\prime} c^{\prime}$ de $\theta$. À l'aide de la formule $\theta$, on peut borner $\operatorname{RM}^{*}(\bar{a} / \bar{b})$ par l'inégalité d'Erimbetov $[6]$.

Fin de la preuve du sous-lemme.

La formule $\varphi \wedge \mathrm{R}(\bar{x})$ a donc un rang de Morley fini dans $\mathrm{DCF}_{0}$ coloré et donc $\operatorname{RM}^{*}\left(p^{*}\right)<\omega$.

Si le RU $(\bar{a} / \bar{b})$ n'est pas monomial, par le fait 3.1 (5), il existe $\bar{c}$ différentiellement algébrique tel que $\mathrm{RU}(\bar{a} / \bar{b} \bar{c})=\omega \cdot n$. Dans le cas non trivial, l'additivité du rang de Lascar implique que $\mathrm{RU}^{*}(\bar{a} / \bar{b})<\omega \cdot 2$, et dans le cas trivial, l'inégalité d'Erimbetov [6] implique que les rangs restent finis. Dans le cas non trivial, une induction du même type que ci-dessus permet en fait de montrer que $\operatorname{RM}^{*}(\bar{a} / \bar{b})<\omega \cdot 2$. De manière plus générale on peut vérifier que pour toute extension autosuffisante $k^{\prime}$ de $k$ de prédimension nulle qui se décompose en une tour finie d'extensions minimales,

$$
\omega \cdot m \leq \mathrm{RU}^{*}\left(k^{\prime} / k\right) \leq \mathrm{RM}^{*}\left(k^{\prime} / k\right)<\omega \cdot(m+1)
$$

où $m$ est le nombre d'extensions correspondantes à des génériques d'espaces affines.

En utilisant cette dernière remarque, le lecteur sagace notera facilement que le type générique rouge est limite de types de rang $\omega \cdot m$ avec $m$ croissant. Il en déduira qu'il a rang $\omega^{2}$. Il notera également que tout blanc est produit de deux rouges et du fait que le rang d'un corps est monomial et que le groupe additif est connexe; il déduira que le type générique blanc est de rang $\omega^{2} \cdot 2$.

D'après la description précédente, pour obtenir un collapse de rang $\omega \cdot 2$, il suffit donc de collapser à rang fini les extensions minimales affines, qu'on appellera préfinitaires. Pour ce faire, nous allons les rendre de dimension finie sur $C$, en leur interdisant d'avoir une infinité de réalisations rouges $C$-linéairement indépendantes. Par la suite nous appelerons extensions finitaires les extensions minimales déterminées par un type trivial 


\section{LES CODES}

Dans cette partie nous travaillons dans $\mathrm{DCF}_{0}$. Nous allons fréquemment adapter les preuves de [3] à ce cadre en utilisant certaines propriétés de la théorie $\mathrm{DCF}_{0}$; et en particulier celles rappelées dans le fait 3.1 et dans la remarque suivante :

Remarque 4.1. Dans $\mathrm{DCF}_{0}$ :

(1) Le générique de $\mathbb{K}^{n}$ est définissable; c'est l'unique type de degré différentiel $n$.

(2) Soient $p$ un type stationnaire de rang de Morley $\omega \cdot n$ et $\bar{e}_{0}, \ldots, \bar{e}_{m}$ une suite de Morley de $p$ au-dessus de $B$. Alors, pour tout $\bar{d}$ de longueur inférieure ou égale à $m$, il existe au moins un $\bar{e}_{i}$ tel que $\bar{d} \underset{B}{\downarrow} \bar{e}_{i}$.

Pour la suite, on dira qu'un ensemble définissable (ou un type) dans $\mathrm{DCF}_{0}$ a un bon rang si son rang de Morley est un monôme de la forme $\omega \cdot n$. Deux ensembles définissables $X$ et $Y$ dans $\mathbb{K}^{n}$ sont dits équivalents si $\operatorname{RM}(X)=\mathrm{RM}(Y)$ et $\operatorname{RM}(X \triangle Y)<\mathrm{RM}(X)$. On notera cette propriété $X \sim Y$. Soit $X$ un sousensemble définissable de $\mathbb{K}^{n}$ qui est génériquement un sous-groupe additif de $\mathbb{K}^{n}$. Son groupe invariant, $\operatorname{Inv}(X)$, est le sous-groupe $\left\{H \in \mathrm{GL}_{\mathrm{n}}(C) \mid H(X) \sim X\right\}$.

Comme tout sous-groupe additif définissable est connexe, tout ensemble définissable $X$, qui est génériquement un sous-groupe additif, est de degré de Morley 1 (c.à.d. "irréductible") et de plus si $X$ a un bon rang alors par la remarque 4.1 (1), le sous-groupe $\operatorname{Inv}(X)$ est définissable.

Le lemme suivant se montre de manière analogue à [3, Lemma 2.7]

Lemme 4.2. Soient $X$ un sous-ensemble B-définissable de $\mathbb{K}^{n}$ ayant un bon rang et de degré de Morley 1 , et $\bar{e}_{0}$ et $\bar{e}_{1}$ deux génériques B-indépendants. Si $\bar{e}_{0}-H \bar{e}_{1} \underset{B}{\downarrow} \bar{e}_{0}$ pour une matrice $H$ de $\mathrm{GL}_{\mathrm{n}}(C)$, alors $X$ est équivalent à un sous-espace affine sur $C$. De plus, si $X$ est équivalent à un sous-espace vectoriel sur $C$, alors $H$ est dans $\operatorname{Inv}(X)$.

Définition 4.3. On appelle ensemble préfnitaire toute partie définissable $X$ de $\mathbb{K}^{2 n}$ à paramètres $\bar{b}$ et de bon rang $\omega \cdot n$ telle que pour tout générique $\bar{a}$ de $X$, les conditions suivantes sont réalisées :

(a) $\operatorname{dim}_{\cdot} \operatorname{lin}_{\mathrm{C}}(\bar{a})=2 n$.

(b) pour toute matrice $M$ de $\mathrm{M}_{2 n}(C)$ de rang $0<d<2 n$,

$$
\operatorname{deg} \cdot \operatorname{dif}(\bar{a} / \bar{b} \cup M \bar{a})<n-d / 2 .
$$

(c) $X$ est équivalent à un sous-espace affine sur $C$.

Notons qu'un ensemble préfinitaire est nécessairement de degré de Morley 1 car tout sous-groupe additif définissable est connexe.

Rappelons que les types de bon rangs ne dévient pas sur les constantes. Ainsi, on peut adapter les arguments utilisés pour les ensembles préalgébriques dans [3, Lemma 3.3], pour vérifier que la préfinitude est conservée par équivalence, translation et action de $\mathrm{GL}_{2 \mathrm{n}}(C)$.

On dira qu'une formule $\varphi(\bar{x}, \bar{y})$ encode un ensemble définissable $X$ si il existe $\bar{b} \in \mathbb{K}$ tel que $X$ et $\varphi(\bar{x}, \bar{b})$ sont équivalents.

Définition 4.4. Un code $\alpha$ est une formule $\varphi_{\alpha}(\bar{x}, \bar{y})$ telle que (pour un entier $n_{\alpha}$ ) : 
(a) La longueur de $\bar{x}$ est $2 n_{\alpha}$.

(b) L'ensemble $\varphi_{\alpha}(\bar{x}, \bar{b})$ est soit vide, soit génériquement un sous-groupe additif de rang $\omega \cdot n_{\alpha}$.

(c) Si $\bar{a}$ est une réalisation de $\varphi_{\alpha}(\bar{x}, \bar{b})$ alors $\bar{a}$ vérifie les conditions (a) et (b) de la définition 4.3 .

(d) Si $\varphi_{\alpha}(\bar{x}, \bar{b})$ et $\varphi_{\alpha}\left(\bar{x}, \bar{b}^{\prime}\right)$ non vides sont équivalents alors $\bar{b}=\bar{b}^{\prime}$.

(e) Pour toute matrice $M \in \mathrm{GL}_{2 \mathrm{n}_{\alpha}}(C)$, l'ensemble $\varphi_{\alpha}(M \cdot \bar{x}, \bar{b})$ est encodé par $\varphi_{\alpha}$.

Rappelons que les rangs de Lascar et de Morley sont les mêmes pour un groupe définissable dans $\mathrm{DCF}_{0}$, il en est donc de même pour un ensemble préfinitaire.

Notons que dans la théorie $\mathrm{DCF}_{0}$ le rang de Morley n'est pas définissable. Afin de montrer que tout groupe préfinitaire peut être codé, nous devons donc vérifier que l'on peut exprimer sur les paramètres qu'un sous-groupe additif a un bon rang.

Lemme 4.5. La propriété d'être un sous-groupe additif $G$ définissable avec un bon rang est définissable sur les paramètres dans le sens suivant : si $\varphi(\bar{x}, \bar{b})$ définit un sous-groupe additif ayant un bon rang $\omega \cdot n$, il existe une formule du type de $\bar{b}$ tel que pour tout $\bar{b}^{\prime}$ satisfaisant cette formule, $\varphi\left(\bar{x}, \bar{b}^{\prime}\right)$ a les mêmes propriétés.

Démonstration. Commençons par remarquer qu'un sous-groupe $G$ définissable de $\mathbb{K}^{m}$ a un bon rang $\omega \cdot n$ si et seulement si il est de degré différentiel supérieur ou égal à $n$ et tout sous-groupe propre définissable est de degré différentiel strictement inférieur à $n$ (voir [13, Théorème 6.7]).

Soit $G$ un sous-groupe additif définissable de $\mathbb{K}^{m}$ avec un bon rang $\omega \cdot n$. Alors $G$ est défini par un système différentiel linéaire $S$ à paramètres $\bar{b}$ que l'on note $S_{\bar{b}}$. Pour exprimer que $S_{\bar{y}}$ est de degré différentiel supérieur ou égal à $n$, il suffit de dire qu'une projection sur $n$ coordonnées est dans le type générique de $\mathbb{K}^{n}$ ce qui est définissable (remarque $4.1(1)$ ).

Fixons un générique $\left(a_{1}, \ldots, a_{m}\right)$ de $G \operatorname{sur} \bar{b}$. Si $a_{i}$ est différentiellement algébrique sur $a_{i_{1}}, \ldots, a_{i_{k}}$ au dessus de $\bar{b}$, on peut supposer que $S_{\bar{b}}$ contient une équation différentielle linéaire l'exprimant et on peut la choisir unitaire en $a_{i}$ (c.à.d. de la forme $\left.a_{i}^{(r)}+\ldots=0\right)$. Ainsi, pour tout paramètre $\bar{y}$, le système $S_{\bar{y}}$ est de degré différentiel inférieur ou égal à $n$.

Il reste maintenant à exprimer que le degré chute dès que l'on considère un sous-groupe propre. Pour un sous-groupe propre donné par une nouvelle équation différentielle linéaire, cela s'exprime sur les paramètres car il suffit de dire qu'une certaine projection n'est pas de degré différentielle maximale. Pour l'exprimer globalement, il suffit de montrer que l'on peut borner l'ordre des équations différentielles à considérer.

Notons $M$ l'ordre de $S_{\bar{y}}$, c'est à dire l'ordre maximal en toutes les variables $x_{1}, . ., x_{m}$. Considérons un sous-groupe défini par $S_{\bar{y}}$ et une seule nouvelle équation différentielle linéaire $g\left(x_{1}, \ldots, x_{k}\right)=0$ (sur des paramètres quelconques). On peut supposer qu'il existe $l \leq k$ tel que $a_{1}, \ldots, a_{l}$ est différentiellement transcendant et $a_{l+1}, \ldots, a_{k}$ différentiellement algébrique sur $a_{1}, \ldots, a_{l}$ au dessus de $\bar{b}$. Si $l=k$, il n'y a rien à dire sur $\bar{y}$, le degré du sous-groupe chute nécessairement. Sinon à l'aide des équations du système, on commence à transformer $g$ tel que l'ordre de $g$ en les variables $x_{l+1}, \ldots, x_{k}$ soit inférieur à $M$.

Dans ce cas nous utiliserons l'argument qui suit, soit pour remplacer $g=0$ par une équation sans la variable $x_{k}$, soit pour borner l'ordre de $g$ : 
Sous-lemme. Soient deux équations différentielles linéaires $g_{0}\left(x_{1}, \ldots, x_{k}\right)=0$ et $f\left(x_{1}, \ldots, x_{k}\right)=0$ d'ordre en $x_{l+1}, \ldots, x_{k}$ borné par $m_{0}$ telles que $f\left(x_{1}, \ldots, x_{k}\right)=0$ a ordre total borné par $M$ et est non trivial en $x_{k}$. Alors le système $g_{0}=0$ et $f=0$ implique un équation différentielle linéaire $g_{1}\left(x_{1}, \ldots, x_{k-1}\right)=0$ telle que l'ordre en $x_{l+1}, \ldots, x_{k-1}$ de $g_{1}$ est borné par $M^{2}+m_{0}$ et l'ordre total de $g_{0}$ est borné par le maximum de l'ordre total de $g_{1}$ et de $M+m_{0}$.

Démonstration. Posons $h_{0}=g_{0}$ et $h_{1}=f$. On commence par construire $h_{2}$ en enlevant à $h_{0}$ les dérivées de $x_{k}$ d'ordre supérieur ou égal à l'ordre de $x_{k}$ dans $h_{1}$ à l'aide de dérivées de $h_{1}$. Alors $h_{2}$ est égal à la somme de $h_{0}$ et d'une combinaison linéaire de dérivées de $h_{1}$, tel que l'ordre de dérivation en $h_{1}$ dans cette expression est au plus $m_{0}$. On en déduit que l'ordre de $h_{2}$ en $x_{l+1}, \ldots, x_{k-1}$ est borné par $M+m_{0}$ et l'ordre de $h_{2}$ en $x_{k}$ est strictement inférieur à $M$. Si $h_{2}$ est non trivial en $x_{k}$, on itère ce procédé avec $h_{1}$ et $h_{2}$, jusqu'à ce que $h_{r}$ soit trivial en $x_{k}$. Ceci se fait en au plus $M$ étapes. Un calcul simple montre alors que l'ordre en $x_{l+1}, \ldots, x_{k-1}$ de $h_{r}$ est borné par $M^{2}+m_{0}$. De plus on vérifie par induction sur $2 \leq i \leq r$ que, $h_{i}$ est égal à une combinaison linéaire de dérivées de $h_{0}$ et de $h_{1}$ tel que dans cette expression, l'ordre de dérivation en $h_{1}$ est au plus égal à $m_{0}$ plus l'ordre de dérivation en $h_{0}$ (on peut remarquer pour cela que l'ordre de dérivation en $h_{0}$ dans cette expression est strictement croissante avec $i$ ). On en déduit que l'ordre total de $g_{0}=h_{0}$ est borné par le maximum de l'ordre total de $h_{r}$ et de $M+m_{0}$. On pose $g_{1}=h_{r}$.

Revenons à notre équation $g=0$. En considérant une équation $f$ de $S$ qui affirme que $x_{k}$ est différentiellement algébrique sur $x_{1}, \ldots, x_{l}$, en posant $g_{0}=g$ et $m_{0}=M$, on obtient une équation $g_{1}\left(x_{1}, \ldots, x_{k-1}\right)=0$ d'ordre en $x_{l+1}, \ldots, x_{k-1}$ borné par $m_{1}=M^{2}+M$. Si cette équation définit elle-même un sous-groupe propre, on traite ce cas par induction sur $k$. Sinon, cette équation est impliquée par le système $S$. Dans ce cas, on itère le procédé de suppression de variables. On obtient une équation $g_{k-l}\left(x_{1}, \ldots, x_{l}\right)=0$ impliquée par $S$, mais alors $g_{k-l}$ est identiquement nul. En utilisant les bornes du sous-lemme précédent, un calcul simple montre que l'ordre total de $g$ est alors borné par $M(2+(k-l-1) M)$.

Lemme 4.6. Tout groupe préfinitaire peut être encodé.

Démonstration. Soit $X$ un groupe préfinitaire défini par $\varphi(\bar{x}, \bar{b})$. Par le lemme 4.5, on peut supposer que $\varphi(\bar{x}, \bar{y})$ satisfasse (b). Les conditions du (c) sont définissables, donc on peut supposer également que $\varphi(\bar{x}, \bar{y})$ les satisfasse. Les conditions (d) et (e) s'obtiennent exactement de la même façon que dans la preuve de [3, Lemma 4.3]. (Notons que $\mathrm{DCF}_{0}$ a élimination d'imaginaires.)

Comme pour les ensembles préalgébriques des corps algébriquement clos de caractéristique fini, on peut choisir un ensemble de bons codes $\mathcal{C}$ tel que tout groupe préfinitaire est encodé par un unique code dans $\mathcal{C}$.

Pour chaque code $\alpha$, on choisit $m_{\alpha}$ tel que pour toute suite de Morley $\bar{e}_{i}$ de $\varphi_{\alpha}(\bar{x}, \bar{b})$ de longueur $m_{\alpha}$ et tout $\bar{b}^{\prime}$ de même taille que $\bar{b}$, alors l'un des $\bar{e}_{i}$ est indépendant de $\bar{b}^{\prime}$ sur $\bar{b}$. (On peut choisir un tel $m_{\alpha}$ plus petit que $|\bar{b}|+2$ par la remarque $4.1(2)$.)

Théorème 4.7. Pour tout code $\alpha$ et tout $\lambda \geq m_{\alpha}$, il y a une formule $\Psi_{\alpha}\left(\bar{x}_{0}, \ldots, \bar{x}_{\lambda}\right)$ telle que : 
(a) Pour toute suite de Morley $\bar{e}_{0}, \ldots, \bar{e}_{\lambda}$ de $\varphi_{\alpha}(\bar{x}, \bar{b})$, on a $\Psi_{\alpha}\left(\bar{e}_{0}, \ldots, \bar{e}_{\lambda}\right)$.

Réciproquement, pour chaque réalisation $\left(\bar{e}_{0}, \ldots, \bar{e}_{\lambda}\right)$ de $\Psi_{\alpha}$ :

(b) Il existe un unique $\bar{b}$ tel que $\models \varphi_{\alpha}\left(\bar{e}_{i}, \bar{b}\right)$ pour $0 \leq i \leq \lambda$. De plus, $\bar{b} \in$ $\operatorname{dcl}\left(\bar{e}_{i_{1}}, \ldots, \bar{e}_{i_{m_{\alpha}}}\right)$ pour tout $i_{1}<\cdots<i_{m_{\alpha}}$. (On appelle $\bar{b}$ le paramètre canonique de la suite $\bar{e}_{0}, \ldots, \bar{e}_{\lambda}$.)

(c) $\bar{e}_{0}, \ldots, \bar{e}_{\lambda}$ sont linéairement indépendants sur $C$.

(d) $\Psi_{\alpha}\left(\bar{f}_{0}, \ldots, \bar{f}_{\lambda}\right)$ pour tout $\left(\begin{array}{c}\bar{f}_{0} \\ \vdots \\ \bar{f}_{\lambda}\end{array}\right)=M\left(\begin{array}{c}\bar{e}_{0} \\ \vdots \\ \bar{e}_{\lambda}\end{array}\right)$ avec $M \in \mathrm{GL}_{\lambda+1}(C)$.

(e) $\Psi_{\alpha}\left(\bar{e}_{0}, \ldots, \bar{e}_{i-1}, \bar{e}_{i}-H \bar{e}_{j}, \bar{e}_{i+1}, \ldots, \bar{e}_{\lambda}\right)$ pour tout $H$ de $\operatorname{Inv}\left(\varphi_{\alpha}(\bar{x}, \bar{b})\right)$ et tout $i \neq j .^{2}$

(f) Si $\bar{e}_{i}$ est générique dans $\varphi_{\alpha}(\bar{x}, \bar{b})$, alors

$$
\bar{e}_{i}-H \bar{e}_{j} \underset{\bar{b}}{\lfloor} \bar{e}_{i}
$$

pour tout $j \neq i$ et $H$ dans $\operatorname{GL}_{2 \mathrm{n}_{\alpha}}(C) \backslash \operatorname{Inv}\left(\varphi_{\alpha}(\bar{x}, \bar{b})\right)$.

Démonstration. Montrons pour commencer que le type partiel suivant vérifie toutes les propriétés voulues :

$$
\begin{aligned}
\Sigma\left(\bar{e}_{0}, \ldots, \bar{e}_{\lambda}\right)= & \quad \text { " } \\
& \text { est existe } \bar{b} \text { tel que suite de Morley de } \bar{e}_{0}, \ldots, \bar{e}_{\lambda}(\bar{x}, \bar{b}) "
\end{aligned}
$$

Par définition, $\Sigma$ satisfait (a) et (c). Le choix de $\bar{m}_{\alpha}$ avec la propriété (d) du code, nous donne (b). Notons que l'uple $\bar{e}_{0} \ldots \bar{e}_{\lambda}$ a un bon rang et est donc indépendant des constantes. On en déduit que toute transformation linéaire de cette suite reste une suite de Morley sur $\bar{b}$ ainsi que la transformation d'un seul élément de la suite par une matrice du sous-groupe invariant associé au code. Par conséquent $\Sigma$ satisfait (d) et (e). Par le lemme 4.2, on a (f).

Par compacité on choisit $\Psi_{0}$ dans $\Sigma$ satisfaisant les propriétés (b), (c) et (f).

On regarde maintenant le groupe $\mathrm{GL}_{\lambda+1}(C)$ comme sous-groupe de $\mathrm{GL}_{2 \mathrm{n}_{\alpha}(\lambda+1)}(C)$ agissant par combinaisons linéaires d'uples de longueur $2 n_{\alpha}$. Le groupe $\mathrm{GL}_{\lambda+1}(C)$ est connexe donc indécomposable dans la structure $C$ qui est un pur corps algébriquement clos. Pour chaque $H \in \mathrm{GL}_{2 \mathrm{n}_{\alpha}}(C)$, soit $C_{H}$ le conjugué de $\mathrm{GL}_{\lambda+1}(C)$ par la matrice

$$
\left(\begin{array}{llll}
H & & & \\
& I d & & \\
& & \ddots & \\
& & & I d
\end{array}\right)
$$

Par le théorème des indécomposables de Zilber, le sous-groupe $G\left(\alpha, \bar{e}_{0}, \ldots, \bar{e}_{\lambda}\right)$ engendré par $\left\{C_{H}\right\}_{H \in \operatorname{Inv}\left(\varphi_{\alpha}(\bar{x}, \bar{b})\right)}$ est uniformément définissable sur $\bar{b}$. Notons que pour tout $H \in \operatorname{Inv}\left(\varphi_{\alpha}(\bar{x}, \bar{b})\right)$, la transformation

$$
\left(\bar{e}_{0}, \ldots, \bar{e}_{i}, \ldots \bar{e}_{\lambda}\right) \rightarrow\left(\bar{e}_{0}, \ldots, \bar{e}_{i-1}, \bar{e}_{i}-H \bar{e}_{j}, \bar{e}_{i+1}, \ldots, \bar{e}_{\lambda}\right)
$$

est dans ce sous-groupe.

\footnotetext{
${ }^{2} \operatorname{Par}(\mathrm{b})$ et $\lambda \geq m_{\alpha}$ cette nouvelle suite a aussi pour paramètre canonique $\bar{b}$.
} 
Soit $\Psi$ définie par

$$
\bigwedge_{\tau \in G\left(\alpha, \bar{e}_{0}, \ldots, \bar{e}_{\lambda}\right)} \Psi_{0}\left(\tau\left(\bar{e}_{0}, \ldots, \bar{e}_{\lambda}\right)\right)
$$

Alors $\Psi$ vérifie toutes les propriétés.

\section{LA CHUTE DES ROUGES}

Dans cette section, on travaille dans le corps différentiel rouge $\mathbb{K}_{\omega}$ (c.f. Théorème 2.1 ) et on considère des structures de $\mathcal{K}_{0}$ plongées de manière autosuffisantes dans $\mathbb{K}_{\omega}$.

Une pseudo-suite de Morley de longueur $\lambda+1$ (avec $\lambda \geq m_{\alpha}$ ) pour le code $\alpha$ est une réalisation rouge d'une formule $\Psi_{\alpha}\left(\bar{x}_{0}, \ldots, \bar{x}_{\lambda}\right)$ satisfaisant les propriétés du théorème 4.7. On appellera transformation d'une pseudo-suite de Morley $\left(\bar{e}_{0}, \ldots, \bar{e}_{\lambda}\right)$, une pseudo-suite obtenue par transformations successives de types (d) ou (e) dans le théorème 4.7. Notons que le paramètre canonique d'une transformation peut être différent de celui de la pseudo-suite de départ.

Lemme 5.1. Pour tout code $\alpha$ et tout entier $r$, il existe une fonction entière $\lambda=$ $\lambda(\alpha, r)$ telle que pour toute extension autosuffisante $k \leq k^{\prime}$ dans $\mathcal{K}_{0}$ vérifiant $C_{k^{\prime}}=$ $C_{k}$ et toute pseudo-suite de Morley $\bar{e}_{0}, \ldots, \bar{e}_{\lambda}$ dans $k^{\prime}$, avec paramètres $\bar{b}$, si la suite ne peut pas être transformée en une pseudo-suite de Morley à paramètres dans $k$, alors on peut extraire une sous-suite de Morley (au sens de $\mathrm{DCF}_{0}$ ) sur $k \cup \bar{b}$ de longueur $r$.

Démonstration. La preuve suit essentiellement celles des articles précédents (voir [9], [3]). Le but est de borner le nombre d'éléments de la suite qui sont des combinaisons linéaires de prédécesseurs modulo $k$. Soit $X$ une partie quelconque de cette suite. Pour chaque $\bar{e}_{i}$ inclus dans le $C_{k^{\prime}}$-espace vectoriel engendré par $X$ au-dessus de $k$, on associe une matrice $H_{i}$ à coefficients dans $C_{k^{\prime}}=C_{k}$ tel que $\bar{e}_{i}=H_{i} X+m_{i}$ avec $m_{i} \in k$. La dimension linéaire $\xi$ de l'espace de telles matrices est bornée en fonction de $|X|$ et $\alpha$. Avec $\xi+1$ tels $\bar{e}_{i}$, on obtient une combinaison linéaire qui chute dans $k$. Par hypothèse le nombre de telles combinaisons linéaires est borné par $m_{\alpha}$ et donc le nombre de $\bar{e}_{i}$ comme ci-dessus est borné en fonction de $|X|$ et $\alpha$. Alors le résultat suit en prenant $X$ minimale contenant $\bar{e}_{0}, \ldots, \bar{e}_{m_{\alpha}-1}$ telle que l'ensemble des coordonnées de la pseudo-suite de Morley est contenu dans le sous-espace vectoriel engendré par $X$ au-dessus de $k$.

De la même façon que dans [3], on choisit deux fonctions $\mu$ et $\mu^{*}$ définies sur l'ensemble de codes $\mathcal{C}$, à valeurs dans $\mathbb{N}$ et à fibres finies telles que :

$-\mu(\alpha) \geq \max \left(\lambda\left(\alpha, \mu^{*}(\alpha)\right), m_{\alpha}\right)$;

$-\mu^{*}(\alpha) \geq \max \left(\lambda\left(\alpha, m_{\alpha}+1\right)+1,2 n_{\alpha}+1\right)$.

et on définit la sous-classe $\mathcal{K}_{\mu}$ des structures dans $\mathcal{K}_{0}$ qui pour tout code $\alpha \in \mathcal{C}$, ne possèdent pas de pseudo-suite de Morley de longueur $\mu(\alpha)+1$.

La classe $\mathcal{K}_{\mu}$ est évidemment élémentaire. (Ajout d'axiomes universels aux axiomes de la classe $\left.\mathcal{K}_{0}\right)$. Nous commençons par vérifier que cette classe a la propriété d'amalgamation.

Les conditions sur les fonctions $\mu$ et $\mu^{*}$ permettent de montrer le lemme suivant en appliquant le lemme 5.1 de la même manière que dans la preuve de $[3$, Lemma 8.1] : 
Lemme 5.2. Soient $k, k_{1}, k_{2} \in \mathcal{K}_{0}$ tels que $k$ est autosuffisant dans $k_{1}$ et $k_{2}$ et tels que $C_{k\left\langle k_{1} \cup k_{2}\right\rangle}=C_{k_{1}}=C_{k_{2}}=C_{k}$. Si pour un code $\alpha \in \mathcal{C}$, l'amalgame libre $k\left\langle k_{1} \cup k_{2}\right\rangle$ contient une pseudo suite de Morley de longueur $\mu(\alpha)+1$ pour le code $\alpha$, alors le paramètre canonique d'une de ses transformations est contenu dans $k_{1}$ ou dans $k_{2}$.

Pour montrer la propriété d'amalgamation pour la classe $\mathcal{K}_{\mu}$, nous allons ajouter des constantes afin de travailler à corps de constantes fixe.

Lemme 5.3. Si $k \in \mathcal{K}_{\mu}$ et $k^{\prime}$ est une extension autosuffisante de $k$ obtenue en ajoutant des constantes alors $k^{\prime} \in \mathcal{K}_{\mu}$.

Démonstration. Soit $\bar{e}_{0}, \ldots, \bar{e}_{\lambda}$ une pseudo-suite de Morley dans $k^{\prime}$ pour le code $\alpha$. Considérons, $\bar{e}_{0}^{\prime}, \ldots, \bar{e}_{\lambda}^{\prime}$ dans $\mathrm{R}(k)$ tel que $\bar{e}_{i}=M_{i} \bar{e}_{i}^{\prime}$ où les $M_{i}$ sont des matrices sur le corps des constantes de $k^{\prime}$. Par conséquent, au sens de $\mathrm{DCF}_{0}$, la clôture différentielle de $k^{\prime}$ satisfait la formule

$\exists M_{0} \ldots, M_{\lambda}$ des matrices sur $C$ de tailles fixées telles que $\Psi_{\alpha}\left(M_{0} \bar{e}_{1}^{\prime}, \ldots, M_{\lambda} \bar{e}_{\lambda}^{\prime}\right)$.

Cette formule est donc satisfaite dans la clôture différentielle de $k$ (qui est sousstructure élémentaire de la clôture différentielle de $k^{\prime}$ ). Du fait que prendre la clôture différentielle n'ajoute pas de constantes, on obtient une pseudo-suite de Morley dans $k$.

Le lemme suivant se montre de manière analogue au [3, Lemma 7.3] (en remarquant tout d'abord, par le lemme précédent, que le corps des constantes est fixe dans ce cas) :

Lemme 5.4. Soient $k \in \mathcal{K}_{\mu}$ et $k^{\prime} \notin \mathcal{K}_{\mu}$ une extension minimale de $k$ contenant une pseudo-suite de Morley $\bar{e}_{0}, \ldots, \bar{e}_{\mu(\alpha)}$ de paramètre canonique $\bar{b} \in k$ pour un code $\alpha \in \mathcal{C}$. Alors il existe une pseudo-suite de Morley $\bar{e}_{0}^{\prime}, \ldots, \bar{e}_{\mu(\alpha)}^{\prime}$ pour le même code et avec même paramètre canonique telle que $k$ contient $\bar{e}_{0}^{\prime}, \ldots, \bar{e}_{\mu(\alpha)-1}^{\prime}$ et $k^{\prime}=k\left\langle\bar{e}_{\mu(\alpha)}^{\prime}\right\rangle$. Notons qu'alors $\alpha$ est l'unique code vérifiant ces propriétés.

Théorème 5.5. La classe $\mathcal{K}_{\mu}$ a la propriété d'amalgamation.

Démonstration. Il suffit de montrer la propriété pour des extensions minimales $k_{1}$ et $k_{2}$ de $k$ dans $\mathcal{K}_{\mu}$. Notons $k^{\prime}$ l'amalgame libre $k\left\langle k_{1} \cup k_{2}\right\rangle$. On peut supposer que $C_{k^{\prime}}=C_{k}$ (il suffit pour cela d'étendre $k$ par les constantes de $k^{\prime}$ en utilisant le lemme 5.3). Avec cette hypothèse, la prédimension est sous-modulaire entre $k$ et $k^{\prime}$ et par conséquent $k^{\prime}$ est une extension minimale de $k_{1}$ et de $k_{2}$. À l'aide des lemmes $5.2,5.4,3.3$ et de la remarque $4.1(2)$, on montre alors, de la même manière que dans la preuve $\mathrm{du}\left[3\right.$, Theorem 8.2], que si $k^{\prime} \notin \mathcal{K}_{\mu}$ alors $k_{1}$ et $k_{2}$ sont isomorphes au-dessus de $k$.

On suit la notation des articles précedents et on dit qu'un corps $K$ dans $\mathcal{K}_{\mu}$ est riche si pour tout sous-corps autosuffisant finiment engendrée $k$ dans $K$ et toute extension finiment engendrée $k \leq k^{\prime}$ dans $\mathcal{K}_{\mu}$, il existe une $k$-copie de $k^{\prime}$ autosuffisante dans $K$. Par la propriété d'amalgamation, il y a un unique corps riche dénombrable (à isomorphisme près). Maintenant, nous vérifions que les corps riches correspondent aux modèles $\omega$-saturés d'une théorie. Pour cela, nous devons commencer par décrire les extensions minimales qui ne restent pas dans la classe $\mathcal{K}_{\mu}$ 
Notons que dans le lemme suivant, on ne suppose pas qu'une extension préfinitaire est engendrée par un type de bon rang dans $\mathrm{DCF}_{0}$ (contrairement aux ensembles préfinitaires qui sont par définition des ensembles définissables ayant bon rang).

Lemme 5.6. Soient $k \in \mathcal{K}_{\mu}$ et $k^{\prime}$ une extension minimale de $k$. Alors :

(1) Ou bien dim. $\operatorname{lin}_{\mathrm{C}}\left(\mathrm{R}\left(k^{\prime}\right) / R(k)\right) \leq 1$ et alors $k^{\prime} \in \mathcal{K}_{\mu}$.

(2) Ou bien $k^{\prime}$ est une extension finitaire ou préfinitaire de $k$, et alors $k^{\prime} \notin \mathcal{K}_{\mu}$ si et seulement si $k^{\prime}$ contient une pseudo-suite de Morley $\bar{e}_{0}, \ldots, \bar{e}_{\mu(\alpha)}$ de paramètre canonique $\bar{b}$ pour un code $\alpha \in \mathcal{C}$ telle que l'une des conditions suivantes soit réalisée :

(a) $k$ contient $\bar{e}_{0}, \ldots, \bar{e}_{\mu(\alpha)-1}$ et $k^{\prime}=k\left\langle\bar{e}_{\mu(\alpha)}\right\rangle$ (dans ce cas $k^{\prime}$ est préfinitaire).

(b) $\mu^{*}(\alpha)$ éléments parmis $\bar{e}_{0}, \ldots, \bar{e}_{\mu(\alpha)}$ forment (au sens de $\mathrm{DCF}_{0}$ ) une suite de Morley de $\varphi_{\alpha}(\bar{x}, \bar{b})$ au-dessus de $k \cup \bar{b}$.

Démonstration. Par le lemme 5.3, on peut supposer que $C_{k^{\prime}}=C_{k}$. Si $k^{\prime}$ est engendré par un point blanc différentiellement algébrique ou générique alors $\mathrm{R}\left(k^{\prime}\right)=$ $\mathrm{R}(k)$ et donc $k^{\prime} \in \mathcal{K}_{\mu}$ car $k^{\prime}$ contient les même pseudo-suites de Morley que $k$.

Sinon, supposons que $k^{\prime}$ contienne une pseudo-suite de Morley $\bar{e}_{0}, \ldots, \bar{e}_{\mu(\alpha)}$ de paramètre canonique $\bar{b}$ pour un code $\alpha \in \mathcal{C}$. On distingue deux cas :

$\mathrm{Ou}$ bien après une transformation de $\bar{e}_{0}, \ldots, \bar{e}_{\mu(\alpha)}$, on peut supposer que $k$ contient $\bar{b}$. Alors par le lemme 5.4 , on est dans le cas (2a) et dans ce cas $k^{\prime}$ est une extension préfinitaire correspondant en fait à un groupe préfinitaire (en particulier cette extension a un bon rang).

Sinon, par le lemme 5.1, au moins $\mu^{*}(\alpha)$ éléments parmis $\bar{e}_{0}, \ldots, \bar{e}_{\mu(\alpha)}$ forment une suite de Morley de $\varphi_{\alpha}(\bar{x}, \bar{b})$ au-dessus de $k \cup \bar{b}$. En particulier, il existe un élément $\bar{e}_{i}$ générique au-dessus de $k \cup \bar{b}$. Ce n'est pas possible si $k^{\prime}$ est engendré par un générique rouge car dans ce cas $\operatorname{dim} \cdot \operatorname{lin}_{\mathrm{C}}\left(\mathrm{R}\left(k^{\prime}\right) / \mathrm{R}(k)\right)=1$.

Notons que le fait qu'une formule $\varphi(\bar{x}, \bar{b})$ de $\mathrm{DCF}_{0}$ vérifie les conditions du lemme 3.3 est définissable sur $\bar{b}$. On appelle formule de delta $n u l$, une formule $\varphi(\bar{x}, \bar{y})$ qui est pour chaque $\bar{b}$, soit vide, soit comme ci-dessus.

Corollaire 5.7. Pour chaque formule de delta nul, $\varphi(\bar{x}, \bar{y})$, il existe un axiome $\forall \exists$, $\chi_{\varphi}$, tel que tout corps $k \in \mathcal{K}_{\mu}$ différentiellement clos satisfait $\chi_{\varphi}$ si et seulement si, pour tout $\bar{b} \in k$ tel qu'il n'y a pas de réalisation rouge de $\varphi(\bar{x}, \bar{b})$ dans $k$, alors aucune extension minimale $k\langle\bar{a}\rangle$, donnée par une réalisation rouge $\bar{a}$ de $\varphi(\bar{x}, \bar{b})$ de degré de différentiel sur $k$ maximal, n'est dans la classe $\mathcal{K}_{\mu}$.

Démonstration. De la même façon que dans la preuve [3, Corollary 7.5], à partir de la caractérisation des extensions minimales qui ne sont pas dans $\mathcal{K}_{\mu}$, en utilisant le lemme 5.6 et le fait que $\mu^{*}$ est à fibres finies, on vérifie facilement qu'il existe une partie finie $\mathcal{C}_{\varphi} \subset \mathcal{C}$ de codes telle qu'une telle extension $k\langle\bar{a}\rangle \notin \mathcal{K}_{\mu}$ si et seulement si $k\langle\bar{a}\rangle$ contient une pseudo-suite de Morley de longueur $\mu(\beta)+1$ pour un certain $\beta \in \mathcal{C}_{\varphi}$

Ainsi, $k$ n'a pas une telle extension minimale dans $\mathcal{K}_{\mu}$ associée à $\varphi(\bar{x}, \bar{b})$ si et seulement s'il existe un code $\beta \in \mathcal{C}_{\varphi}$, des uples rouges $\bar{m}_{0}, \ldots, \bar{m}_{\mu(\beta)}$ de $k$ et des matrices $M_{0}, \ldots, M_{\mu(\beta)}$ à coefficients dans $C_{k}$ tels que le degré différentiel de la différence symétrique de $\varphi(\bar{x}, \bar{b})$ et $\Psi_{\beta}\left(M_{0} \cdot \bar{x}+\bar{m}_{0}, \ldots, M_{\mu(\beta)} \cdot \bar{x}+\bar{m}_{\mu(\beta)}\right)$ chute. C'est une propriété uniformément définissable en les paramètres (par définissabilité 
du générique de $\mathrm{DCF}_{0}$ ). De plus, en utilisant l'élimination des quantificateurs dans $\mathrm{DCF}_{0}$, on remarque que cette propriété est définissable par une formule $\forall \exists$.

Considérons maintenant la théorie $T^{\mu}$ suivante :

(1) Axiomatisation de $\mathcal{K}_{\mu}$;

(2) Axiomatisation de $\mathrm{DCF}_{0}$;

(3) Les axiomes $\chi_{\varphi}$ pour toute formule $\varphi$ de delta nul.

Théorème 5.8. Un corps $K$ de $\mathcal{K}_{\mu}$ est riche si et seulement si $K$ est un modèle $\omega$-saturé de $T^{\mu}$.

Démonstration. Montrons qu'un modèle $K \omega$-saturé est riche. Soit $k$ finiment engendré tel que $k \leq K$ et $k^{\prime}$ une extension minimale de $k$ dans $\mathcal{K}_{\mu}$.

Si $k^{\prime}$ est engendré au-dessus de $k$ par un élément différentiellement algébrique blanc alors il suffit de réaliser le type corpique de cet élément dans $K$ ce qui est possible car $K$ est un modèle $\omega$-saturé de $\mathrm{DCF}_{0}$.

Si $\delta\left(k^{\prime} / k\right)=0$ et $k^{\prime}$ est engendré par une base rouge $\bar{a}$ de taille $2 n$. On peut supposer que $\bar{a}$ est en amalgame libre avec $K$ au-dessus de $k$. Par saturation, il suffit de réaliser dans $K$ toute formule de delta nul du type rouge de $\bar{a}$ sur $k$. Si une telle formule n'était pas réalisé, alors par l'axiome $\chi_{\varphi}$, l'amalgame libre de $K$ et $\bar{a}$ ne serait pas dans la classe $\mathcal{K}_{\mu}$, ce qui contredirait la propriété d'amalgamation.

Si $k^{\prime}$ est engendré par le générique rouge au-dessus de $k$, on l'approxime dans $K$ par des types rouges préfinitaires donnés par $\left(a, a^{(n)}\right)$ (qui sont dans $\mathcal{K}_{\mu}$ car de dimension linéaire deux) et donc par saturation, ce générique est réalisé dans $K$. Le même argument d'approximation montre que $K$ réalise le générique blanc.

Montrons maintenant que les corps riches sont modèles de $T^{\mu}$. Soit $K$ un corps riche. Alors $K$ est bien dans $\mathcal{K}_{\mu}$ et il est différentiellement clos car tout type différentiellement algébrique sur un ensemble fini est réalisé par un point blanc dans $K$. (Il suffit de plonger l'amalgame libre d'une extension par un point différentiellement algébrique).

Soit $\varphi(\bar{x}, \bar{b})$ une formule de delta nul avec $\bar{b} \in K$. Soit $k$ contenant $\bar{b}$, corps finiment engendré et autosuffisant dans $K$. S'il existe une réalisation rouge $\bar{a}$ de $\varphi(\bar{x}, \bar{b})$ telle que $k\langle\bar{a}\rangle$ est dans $\mathcal{K}_{\mu}$, alors par richesse il y a une réalisation de $\varphi(\bar{x}, \bar{b})$ dans $K$. Sinon, la seconde partie de l'axiome est satisfaite.

Puisque tous les riches se correspondent par va et vient infini, on déduit qu'ils sont tous $\omega$-saturés.

Pour la suite, nous notons $K$ l'unique modèle riche dénombrable. Remarquons qu'il n'y a pas d'extension préfinitaire au-dessus de $K$ dans $\mathcal{K}_{\mu}$ : sinon à partir d'une réalisation rouge générique d'une cossette d'un groupe codé par $\alpha \in \mathcal{C}$, on pourrait facilement trouver dans $K$ une suite de Morley rouge de ce groupe, de longueur arbitrairement grande, et obtiendrait, par la propriété (a) de $\Psi_{\alpha}$, une pseudo-suite de Morley de longueur trop grande.

Corollaire 5.9. La théorie $T^{\mu}$ est de rang $\omega \cdot 2$ et les rouges forment un sous-groupe additif de rang $\omega$.

Démonstration. Soit $k^{\prime}$ une extension minimale affine de bon rang sur $k$ engendrée par une base rouge $\bar{a}$. Rappelons que le type de $\bar{a}$ est alors le générique d'une cossette définissable d'un groupe codé par $\varphi_{\alpha}(\bar{x}, \bar{b})$, où l'on peut supposer $\bar{b}$ autosuffisant. Si on pouvait trouver une suite assez longue des réalisations de $\varphi_{\alpha}(\bar{x}, \bar{b})$ non 
complètement lineairement dépendantes, on obtiendrait une suite de Morley (car la prédimension ne chute pas indéfiniment). Du fait que la taille des pseudo-suites de Morley correspondantes est bornée, l'espace de réalisations rouges de $\varphi_{\alpha}(\bar{x}, \bar{b})$ est finiment engendré sur les constants. Comme $C$ est de rang 1, le rang de Morley de $\varphi_{\alpha}(\bar{x}, \bar{b}) \wedge \mathrm{R}(\bar{x})$ est donc fini (borné indépendemment de $\bar{b}$ ).

Les extensions minimales de prédimension nulle sont donc toutes de rangs finis. Le même calcul de rangs que dans le sous-lemme de 3.4 nous permet de borner uniformement le rang de chaque morceau dans une tour d'extensions minimales de prédimension nulle et à nouveau grâce à l'inégalité d'Erimbetov, on conclut que toute les extensions autosuffisantes de prédimension nulle ont rang de Morley fini. Donc le générique rouge est de rang au plus $\omega$.

Tout point est produit de deux rouges : en effet, pour $a \in K$, on considère une clôture autosuffisante $k$ de $a$ et on prend une paire de rouges $r_{1}, r_{2}$ linéairement indépendants sur $k$ telle que $a=r_{1} \cdot r_{2}$ et $r_{1}$ est différentiellement transcendant sur $k$. Cette paire détermine alors une extension triviale qui est donc réalisée dans $K$ (qui sont dans $\mathcal{K}_{\mu}$ car de dimension linéaire deux par le lemme 5.6). Comme $K$ ne peut pas avoir rang fini, on en déduit que le générique rouge est de rang $\omega$ et par connexité du groupe additif de $K$, le rang du générique blanc est $\omega \cdot 2$.

\section{LES VERTS}

Dans cette dernière section, nous travaillons dans le modèle riche $K$ obtenu dans section 4.

Considérons le logarithme différentiel défini de la façon suivante :

$$
\begin{aligned}
\mathfrak{L} \partial: \mathbb{G}_{m} & \rightarrow \mathbb{G}_{a} \\
x & \rightarrow \frac{x^{\prime}}{x}
\end{aligned}
$$

où $\mathbb{G}_{m}$ (resp. $\left.\mathbb{G}_{a}\right)$ dénote le groupe algébrique multiplicatif (resp. additif) de $K$. Notons que $\mathfrak{L} \partial$ est un homomorphisme de groupes avec noyau $\mathbb{G}_{m}(C)=C^{*}$. On défini maintenant :

$$
\mathrm{V}=\left\{\bar{x} \in \mathbb{G}_{m} \mid \mathfrak{L} \partial(x) \in \mathrm{R}\right\}
$$

et on l'appelle ensemble de points verts de $K$. Il est un sous-groupe multiplicatif divisible (parce que $\mathrm{R}$ est un $\mathbb{Q}$-espace vectoriel) qui contient de la torsion (toute la torsion de $\left.\mathcal{C}^{*}\right)$. Poizat [14] avait déjà noté que la structure réduite $(K, \mathrm{~V})$ n'est pas un mauvais corps, même si on oublie la dérivé (et donc les rouges). Nous répétons et complétons maintenant son argument. Dans le langage étendu, $\mathrm{V}$ a aussi rang $\omega$ (parce que le noyau de $\mathfrak{L} \partial$ a rang 1 et l'application $\mathfrak{L} \partial$ est surjective car $K$ est différentiellement clos). De plus, $\mathrm{V}$ est divisible, et donc indécomposable (multiplicativement mais aussi additivement). Le théorème des indécomposables [13, Théorème 6.10] montre qu'il existe une borne $\xi$ (en fait, 4) telle que tout élément de $K$ s'exprime comme somme de $\xi$ éléments de $V$. Cet énoncé reste alors vrai dans la structure réduite, donc $K$ et $\mathrm{V}$ ont des rangs commensurables dans la structure $(K, \mathrm{~V})$. Maintenant, [15] donne l'existence d'un plus petit corps définissable $F$ contenant le corps de constantes $C$. En passant au langage étendu, on déduit que $F$ a rang fini (même en passant au langage étendu car $F$ est strictement contenu dans $V \cup\{0\}$ ), et donc $F=C$ parce que $C$ est algébriquement clos. Ainsi le rang de $C$ dans le réduit reste 1 , et le rang de $K$ est au moins $\omega$. Par commensurabilité 
de $K$ et $\mathrm{V}$ et le fait que $K$ est multiplicativement connexe, le rang de $\mathrm{V}$ reste $\omega$ et le rang de $K$ est $\omega \cdot 2$ dans la structure réduite $(K, V)$.

En utilisant l'application de Manin, on obtient des sous-groupes non-algébriques d'une variété semiabelienne donnée telles que la structure réduite n'a pas rang fini.

\section{RÉFÉRENCES}

[1] A. Baudisch, M. Hils, A. Martin-Pizarro, F. Wagner, Die böse Farbe, soumis en 2006.

[2] A. Baudisch, A. Martin-Pizarro, M. Ziegler, On fields and Colours, Algebra i Logika, 45, $\mathrm{n}^{\circ}$ 2, 92-105 (2006).

[3] A. Baudisch, A. Martin-Pizarro, M. Ziegler, Red fields, J. Symbolic Logic, 72, vol. 1, 207-225, 2007.

[4] F. Benoist Rangs et types de rang maximum dans les corps différentiellement clos, J. Symbolic Logic, 67, vol 3, 1178-1188, (2002).

[5] E. Bouscaren, Model Theory and Algebraic Geometry : an introduction to E. Hrushovski's proof of the geometric Mordell-Lang conjecture, Lecture Notes in Mathematics, 1696, Springer-Verlag, Berlin, Germany, 1991.

[6] M. Erimbetov, Complete theories with 1-cardinal formulas, Algebra i Logika, 14, no $3,245-$ $257,(1975)$.

[7] E. Hrushovski, The Mordell-Lang conjecture for function fields, J. Amer. Math. Soc.,9, $\mathrm{n}^{\circ}$ 3, 667-690, 1996.

[8] E. Hrushovski, Strongly minimal expansions of algebraically closed fields, Israel J. Math, 79 , 129-151, (1992).

[9] E. Hrushovski, A new strongly minimal set, Annals of Pure and Applied Logic, 62, 147-166, (1993).

[10] D. Marker, Model theory of differential fields, dans Model theory of fields, Lecture Notes in Logic, 5, ASL, La Jolla(CA), EU, (1996).

[11] A. Pillay, Geometric Stability Theory, Oxford Logic Guides, 33. Oxford University Press, New York, (1996).

[12] A. Pillay, W.Y. Pong, On Lascar rank and Morley rank of definable groups in differentially closed fields, J. Symbolic Logic, 67, vol 3, 1189-1196, (2002).

[13] B. Poizat, Groupes Stables. Une tentative de conciliation entre la géométrie algébrique et la logique mathématique, Nur al-Mantiq wal-Ma'rifah, Bruno Poizat, Lyon (1987).

[14] B. Poizat, L'égalité au cube, J. Symb. Logic, 66, no 4, 1647-1676, (2001).

[15] F.O. Wagner, Subgroups of stable groups, J. Symb. Logic, 55, no 1, 151-156, (1990).

[16] F.O. Wagner, Bad fields in positive characteristic, Bull. London Math. Soc, 35, $\mathrm{n}^{\circ}$ 4, 499-502, (2003).

[17] C. Wood, Differentialy closed fields In E. Bouscaren, editor, Model Theory and Algebraic Geometry, Lecture Notes in Mathematics 1696. Springer-Verlag, (1998).

[18] M. Ziegler, Lemma für Daniels beschränkte Automorphismen, preprint at http ://de.arxiv.org/math.LO/0608433

T. Blossier, Université de Lyon, Université Lyon 1, Institut Camille Jordan UMR 5208 CNRS, 43 Boulevard du 11 novembre 1918, F-69622 Villeurbanne Cedex, France

E-mail address: blossier@math.univ-lyon1.fr

A. Martin Pizarro, Institut für Mathematik, Humboldt-Universität Zu Berlin, D10099 Berlin, Allemagne.

Adresse actuelle : Université de Lyon, Université Lyon 1, Institut Camille Jordan UMR 5208 CNRS, 43 boulevard du 11 novembre 1918, F-69622 Villeurbanne Cedex, France

E-mail address: pizarro@mathematik.hu-berlin.de, pizarro@math.univ-lyon1.fr 